\title{
La formación de alumnado con discapacidad visual en el marco de los conservatorios de música en España
}

\author{
Luis Sánchez Sánchez'; Práxedes Muñoz Sánchez²
}

Recibido: 1 de diciembre de 2019 / Aceptado: 20 de abril de 2020

Resumen. El presente artículo informa sobre la investigación realizada entre 2013 y 2017 acerca de la enseñanza de alumnado con discapacidad visual en los Conservatorios de Música, recogiendo los recursos y buenas prácticas más destacadas que se revelaron sobre el fomento de la inclusión para estos centros, siendo también muchas de ellas extrapolables a las escuelas de música y/o las agrupaciones musicales que integran ciegos. Se parte de las dificultades de la temática en la reducida bibliografía y que el profesorado de música no cuenta con la suficiente información, experiencia y formación previa para instruir a estudiantes invidentes (Chaves, Godall y Zattera, 2015; Chávez, 2010; Dias, 2010). La metodología de la investigación fue la etnografía a partir de un diálogo de saberes entre alumnado con discapacidad visual, sus familias y docentes junto a especialistas de este campo. Para la evaluación de los resultados se conectó con la metodología del "juicio de expertos", que evaluaron las estrategias y los recursos. Finalmente esta investigación visibiliza las prácticas que son útiles para el desarrollo de una educación inclusiva musical para alumnado con discapacidad visual.

Palabras clave: educación especial; música y musicología; etnografía y etnología; metodología; pedagogía.

\section{[en] The training of students with visual disabilities within the framework of music conservatories in Spain}

Abstract. This article reports on the research carried out between 2013 and 2017 on the teaching of students with visual disabilities at the Conservatories of Music, gathering the most outstanding resources and best practices that were revealed on promoting inclusion for these centers, also being many of them can be extrapolated to music schools and / or musical groups that make up the blind. It starts from the difficulties of the subject in the reduced bibliography and that the music teachers don't have enough information, experience and previous training to instruct blind students (Chaves, Godall and Zattera, 2015; Chávez, 2010; Dias, 2010). The research methodology was ethnography based on a dialogue of knowledge between visually impaired students, their families and teachers together with specialists in this field. For the evaluation of the results, it was connected with the "expert judgment" methodology, which evaluated the strategies and resources. Finally, this research makes visible the practices that are useful for the development of an inclusive musical education for students with visual disabilities.

Keywords: special education; music and musicology; ethnography and ethnology; methodology; pedagogy.

Sumario. 1. Introducción. 2. Objetivos. 3. Método. 4. Resultados de la recopilación para la enseñanza musical del alumnado con ceguera. 5. Análisis y evaluación de los resultados. 6. Conclusiones. 7. Referencias bibliográficas.

Cómo citar: Sánchez, L. y Muñoz, P. (2020). La formación de alumnado con discapacidad visual en el marco de los conservatorios de música en España, en Revista Electrónica Complutense de Investigación en Educación Musical, 17, 49-61

\footnotetext{
Consellería de Educación de la Generalitat Valenciana

E-mail: sanchez_luisana@gva.es

2 Universidad Católica de Murcia (España)

E-mail: pmunoz@ucam.edu
} 


\section{Introducción}

El alumnado con ceguera es una realidad en los centros educativos, ya que siempre que es adecuado y posible, la atención a estos estudiantes debe efectuarse en centros ordinarios (Albertí y Romero, 2010; Garaialde, Laquidain y Urbina, 1992). Por ello, esta investigación tuvo desde su origen dos objetivos básicos que la justifican:

- Por un lado, resolver las dudas que pueden tener los docentes de conservatorio sobre cómo enseñar al alumnado que presenta ceguera.

- Por otro, elaborar alguna herramienta o método que ayudara en esta labor.

Todo desde la visión que la discapacidad visual pueda ser una fuente de enriquecimiento para el docente, tanto a nivel didáctico al poder integrar algunos de esos recursos, prácticas y estrategias con el resto de alumnado, como también a nivel personal, tal y como apuntan Acero (2010), Espejo (1993) y Tomás y Barcelón (2006) a raíz de las experiencias inclusivas que desarrollaron. De esta forma, no entendemos la discapacidad como una "barrera", la cual puede salvarse en la enseñanza (Quintero, 2000), sino como más bien una "oportunidad" de crecimiento.

Conviene tener en cuenta que en lo que concierne a la educación musical del alumnado con discapacidad visual, las nuevas tecnologías ofrecen oportunidades para proporcionar y recibir información (Gorbunova y Voronov, 2018), aspecto que durante la investigación se conoció y en los resultados se abordan al concretar diversas herramientas para este ámbito.

Sin embargo, tres problemas fundamentales se encontraron en esta investigación: por un lado, la escasa bibliografía existente en este campo; por otro lado, el hecho de que habitualmente los docentes de música se encuentran desorientados para enseñar a estudiantes invidentes; y finalmente, que hay una carencia de información en esta área (Chávez, 2010; Dias, 2010; Chaves et al., 2015), como se aprecia en Chaves et al. (2015):

Hay que resaltar que muchos de los educadores no están preparados para recibir alumnos con discapacidad visual en las clases de música. El profesor que no tiene experiencia o formación, se encuentra con innumerables dudas e incertidumbres acerca de cómo enseñar a una persona con discapacidad visual, muchas de las dudas están relacionadas con el funcionamiento del sistema de escritura musical en Braille, el acceso a materiales didácticos transcritos o adaptados, las estrategias de enseñanza que se deben utilizar en clase, la comunicación con el alumno, etc. (Chaves et al., 2015, p. 149).

A nivel sociocultural se debe también tener presente que en 1993 había 16 agrupaciones musicales con ciegos en España (Díez, 1996). Sin embargo, en la actualidad, sólo hay 5 agrupaciones musicales con invidentes en nuestro país, tal y como el trabajo de campo reveló; una de cuerda, en este caso la Orquesta de Plectro "Ciudad de los Califas" de Córdoba y cuatro corales: Coral "Allegro" de Valencia, Coral "Alaia" de San Sebastián, Orfeón "Fermín Gurbindo" de Madrid y Coral "Cidade de Vigo".

Si bien, la investigación abordó la enseñanza del conservatorio de música, en general, y el presentar herramientas para el conjunto de asignaturas de las enseñanzas elementales y profesionales de música, se optó en lo relativo al instrumento especificarlas para el violín, instrumento que tocamos y que el trabajo de campo reveló que hay pocos estudiantes ciegos.

De ahí surgieron las preguntas de investigación:

- ¿Puede aportarse alguna herramienta que potencie la inclusión en el aula de violín?

- ¿Es preciso un programa específico para este alumnado con ceguera?

- ¿Los conservatorios y su normativa están preparados para atender a este alumnado?

- ¿Es necesaria una formación específica para el profesorado?

Estas preguntas de investigación tenían en cuenta dos dificultades añadidas que el violín así como otros instrumentos de cuerda-arco presentan:

- El escaso alumnado con discapacidad visual en los instrumentos de cuerda-arco.

- Los obstáculos concretos que presenta el aprendizaje del violín en los ciegos, entre los cuales podemos enumerar los obstáculos para incorporarse en una orquesta, la necesidad de memorizar todo el repertorio y los movimientos de arco, el estudio de la técnica en la mano derecha, la posible escasa empatía del profesor, la imposibilidad de realizar la práctica de primera vista o las dificultades que tiene la musicografía braille, entre otras.

Todas estas cuestiones, se recogieron gracias a la etnografía en los escenarios de diversos centros/organismos y a los protagonistas de esta área, no solo del ámbito nacional sino también del extranjero: especialistas en el código musicográfico braille, especialistas de los Centros de Recursos Educativos de la ONCE, directores de agrupaciones que integran ciegos tanto de España como del extranjero, profesores de alumnado con discapacidad visual, especialistas 
del ámbito que han creado recursos, dispositivos o dirigen proyectos sobre esta temática, músicos ciegos, alumnado de conservatorio con discapacidad visual y sus familiares, así como especialistas y profesionales diversos.

Sirva de ejemplo estos dos testimonios recogidos en entrevistas realizadas para este trabajo al prestigioso violinista japonés ciego de nacimiento Takayoshi Wanami, y al maestro José Luis Cladera, director de la Banda Sinfónica Nacional de Ciegos "Pascual Grisolía" de Argentina entre 2011 y 2017, que confirman algunas de las dificultades recopiladas anteriormente:

Es extremadamente difícil para un instrumentista ciego trabajar como músico de cámara o de orquesta, debido a que debe memorizar cada nota para tocar (T. Wanami, entrevista, 16/03/2017).

La mayor dificultad a vencer es el manejo de la mano derecha. Del arco propiamente dicho. Su estudio habitual frente al espejo, según me cuentan algunos docentes de contrabajo, no es posible en la persona ciega -claro está- y sumado a la imposibilidad de chequear la manera de desplazar el arco por las cuerdas, paralelo y algo inclinado al puente y demás, hacen la cosa mucho más compleja que en instrumentos de viento en donde todo es fijo (J. L. Cladera, entrevista, 01/10/2015).

Por otro lado, si bien en estos estudios concretos del conservatorio de música hay poco explorado en lo que se refiere a la atención de alumnos ciegos, si es mucho mayor la bibliografía e información sobre la discapacidad visual y la atención escolar de este alumnado. Por ello, se utilizó algunas bases de datos específicas de ésta área, como es el caso del Catálogo de Publicaciones de Servicios Sociales de la Organización Nacional de Ciegos Españoles (ONCE), que contiene un amplio fondo bibliográfico sobre discapacidad visual. De esta forma, se conoció cómo es la enseñanza general de estos estudiantes (la cual no olvidemos que la compaginan con sus estudios de conservatorio) y se trató de adaptar algunas de esas estrategias a la enseñanza del conservatorio; teniendo en cuenta recursos tanto para ciegos totales como para estudiantes con resto visual, pues la enseñanza a alumnos con visión limitada es distinta a la de los alumnos con ceguera total (Barraga, 1985; Gorbunova y Voronov, 2018).

Por último, otros estudios realizados con estudiantes ciegos (Abramo y Pierce, 2013), consideraron la importancia de que la enseñanza debe ser diferente a alumnado con la discapacidad visual de quien no lo tiene, esta etnografía fue con alumnado ciego en una escuela pública. Esto puede depender de los objetivos de la enseñanza. En nuestra investigación observamos que hay determinadas asignaturas que no requieren esta diferenciación, además, mucho de este alumnado no tiene o tiene poco conocimiento del sistema braille.

\section{Objetivos}

La investigación se centró en ofrecer respuestas a estos problemas y preguntas de investigación, teniendo como objetivo general facilitar la enseñanza de violín en el alumnado con deficiencia visual de los Conservatorios de Música, elaborando una herramienta que facilitara su enseñanza (Sánchez, 2017).

Para lograr el objetivo principal se siguieron estos objetivos del proceso de investigación (Sánchez, 2017):

- Elaborar un marco teórico que abordara diversos aspectos de esta área como la discapacidad visual, el sistema braille, la ONCE, la enseñanza escolar con discapacitados visuales, el panorama actual y tendencias de la didáctica musical en España y en el extranjero o la musicografía braille, entre otros.

- Desarrollar un trabajo de campo para investigar sobre las necesidades de este alumnado y músicos invidentes, los recursos y estrategias que utilizan, adentrándonos en los Centros de Recursos Educativos (CREs) de la ONCE, las agrupaciones musicales que integran deficientes visuales en España y en el extranjero, los proyectos realizados y las dificultades que presenta este alumnado.

- Realizar un compendio de buenas prácticas, recursos y estrategias metodológicas para las enseñanzas elementales y profesionales de música. Este objetivo fue concretándose con el transcurso de la investigación como posteriormente se verá en los resultados, la discusión y las conclusiones.

- Evaluar las diferentes estrategias y recursos planteados a través de un análisis del compendio elaborado por parte de diversos especialistas, permitiendo así, resolver algunos detalles a partir de sus sugerencias y valoraciones.

\section{Método}

De acuerdo con Taylor y Bogdan (1987) este trabajo ha seguido una metodología cualitativa, con la etnografía como piedra angular del mismo, focalizada hacia una educación reflexiva y trabajo colaborativo a través de un diálogo de saberes (Dietz, 2011; Dietz, 2012; Muñoz, Mateo y Álvarez, 2014; Vitón, 2012), junto a la experiencia en la docencia a discapacitados visuales, añadiendo las tres dimensiones que estima Dietz (2009) con el fin de lograr una plena etnografía reflexiva: dimensión semántica (entrevistas etnográficas), dimensión pragmática (observaciones participantes) y dimensión sintáctica (diálogo de saberes); fundamental en las experiencias etnográficas ya que permiten realizar una metodología colaborativa para encontrar respuestas más holísticas (Muñoz, 2012). 
Asimismo, y tomando como referentes a Aguilar y Barroso (2015), Arias (2000), Betrián, Galitó, García, Jové y Macarulla (2013), Donolo (2009) y Rodríguez, Pozo y Gutiérrez (2006), se utilizó la triangulación de datos a nivel de participantes (individuos, familias y colectivos) y de métodos (entrevista en profundidad, observación participante y no participante y cuestionario de evaluación), organizado como datos multirreferenciales (Velasco y Díaz de Rada, 1997). La observación y la entrevista fueron los dos instrumentos de recogida de datos fundamentales en la investigación.

Todo ello, desembocó en un "aprendizaje-servicio" entre el investigador, docentes involucrados, alumnado en general y el colectivo de personas con discapacidad visual, al aglutinar el aprendizaje curricular con el servicio a la comunidad (Campo, 2008; Folgueiras, Luna y Puig, 2013; Lucas y Martínez, 2012; Mendía, 2012; Puig, Gijón, Martín y Rubio, 2011; Tapia, 2010).

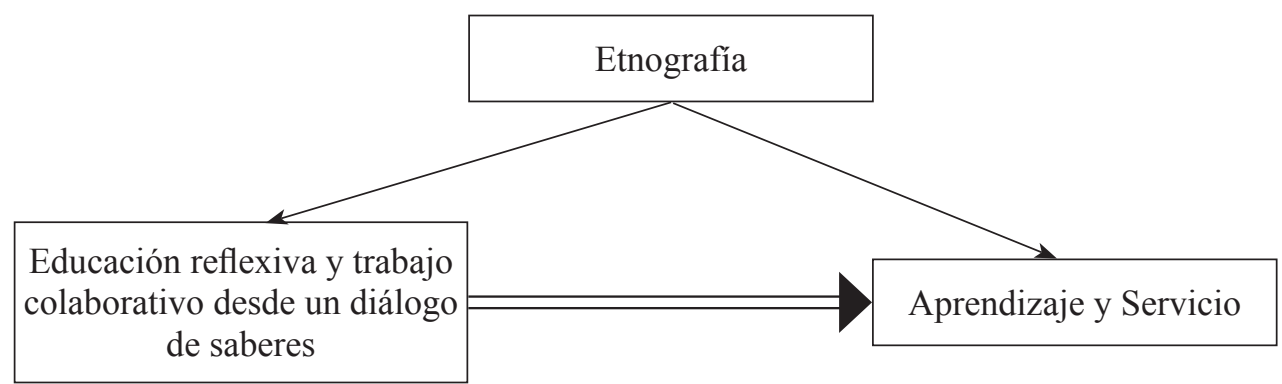

Figura 1. Metodologías que fundamentaron la investigación

Fuente: elaboración propia

A continuación se presenta una categorización de los perfiles de los entrevistados.

Tabla 1. Categorías consideradas de los diversos perfiles de los entrevistados

\begin{tabular}{|l|l|}
\hline $\mathbf{N}^{\mathbf{0}}$ & \multicolumn{1}{|c|}{ CATEGORÍA } \\
\hline 1 & Especialistas de música de los CREs de la ONCE \\
\hline 2 & Especialistas en el código musicográfico braille \\
\hline 3 & $\begin{array}{l}\text { Directores o dirigentes de agrupaciones musicales del extranjero que } \\
\text { integran ciegos }\end{array}$ \\
\hline 4 & Directores de agrupaciones musicales de España que integran ciegos \\
\hline 5 & $\begin{array}{l}\text { Profesores que han atendido o atienden alumnado con discapacidad } \\
\text { visual }\end{array}$ \\
\hline 6 & $\begin{array}{l}\text { Especialistas del ámbito universitario que han creado recursos, dispo- } \\
\text { sitivos o dirigen proyectos }\end{array}$ \\
\hline 7 & Músicos con discapacidad visual \\
\hline 8 & Especialistas y profesionales diversos \\
\hline 9 & Alumnado de conservatorio con discapacidad visual \\
\hline 10 & Familiares de alumnado de conservatorio con deficiencias visuales \\
\hline
\end{tabular}

Fuente: elaboración propia

Para evaluar los resultados obtenidos, se contó con cinco expertos que, a través de un cuestionario y tablas de valoración (Sánchez, 2017), evaluaron las diferentes partes que integra el compendio, conectando así con la metodología del "juicio de expertos" (Cabero y Llorente, 2013; Escobar-Pérez y Cuervo-Martínez, 2008; Robles y Rojas, 2015).

Entre los criterios para la selección de estos expertos se consideraron los siguientes: que hubiese varios especialistas ciegos, perfiles variados, profesionales con interés en esta área, representación de la ONCE, algún especialista del extranjero, algún director con experiencia en atender músicos y/o coralistas invidentes, un especialista en musicografía braille y un profesor de violín que atienda a alumnado con discapacidad visual. De esta manera, se pretendía visualizar y analizar el compendio de estrategias de enseñanza-aprendizaje y las diversas asignaturas que integraba. Los expertos con los que se contó fueron:

- L. C.: profesora de lenguaje musical (jubilada) del Conservatorio Superior de Música "Manuel de Falla" de Buenos Aires. 
- M. C.: corrector de partituras de la Imprenta Braille de la ONCE en Madrid, pianista y profesor de música de cámara.

- C. F.: directora del Orfeón "Fermín Gurbindo" de Madrid, la cual tiene en su agrupación a coralistas ciegos.

- M. J. G.: profesor de violín del Conservatorio Profesional de Música "Guitarrista José Tomás” de Alicante; en el momento de la investigación atendía a una alumna con discapacidad visual.

- M. A. M., especialista de música del CRE "Luis Braille" de Sevilla de la ONCE.

\section{Resultados de la recopilación para la enseñanza musical del alumnado con ceguera}

Se comprobó que este alumnado no precisa un programa distinto al de los videntes, sino básicamente adaptaciones a nivel de metodología, tal y como exponen tanto en el ámbito musical como escolar numerosos autores (Clark y Murphy, 1999; Arnaiz, 1994; Fuentes,1995; Marcos, 1999; Acero, 2010; Tomás y Barcelón, 2006; González, Martínez, Fernández, Soler y Ruíz, 2000). Por ello, la herramienta inclusiva que el objetivo general de la investigación consideraba, inicialmente en crear un repertorio o método propio, posteriormente un proyecto educativo, y finalmente, desemboca en el planteamiento final de, a partir de los resultados, elaborar un compendio de buenas prácticas, recursos y estrategias metodológicas. Estos resultados obtenidos, fueron fruto de la etnografía desde el estudio y revisión de las mismas, y su posterior análisis y adaptación para los estudios en el Conservatorio de Música para teorizar a partir de la práctica.

Sería imposible concretar todas las estrategias, recursos y buenas prácticas, pero para poder apreciar la dimensión del trabajo, hacemos un recorrido por todas las asignaturas que el compendio aborda, de acuerdo con el currículo de las enseñanzas elementales y profesionales de música, y se recopilan las más destacadas ${ }^{3}$ :

Para una mejor lectura de los datos, dividimos los mismos en asignaturas de las dos primeras etapas en las enseñanzas de la música que considera el currículo (elemental y profesional) y que esta investigación abordó.

\subsection{Enseñanzas elementales: asignatura de violín}

A nivel de la enseñanza elemental del violín concretamos por áreas algunas de las estrategias y recursos más relevantes:

- Actitud y relación con el alumno: naturalidad, entender sus dificultades y adecuarnos a sus necesidades.

- Ayuda y colaboración interna y externa: apoyo del especialista del CRE de la ONCE y colaboración de las familias:

Yo tuve clases de violín privadas hasta los 18 años, y mi madre estaba siempre en las lecciones, observando con cuidado para así repetir en casa. Mi ventaja fue que mi madre no era músico, por lo que ella veía las lecciones con sumo cuidado, y no añadió ninguna cuestión innecesaria al repetir las lecciones en casa (T. Wanami, entrevista, 07/01/2016).

- Elementos y recursos materiales: musicografía braille, braille'n speak o programa informático BME.

- Estrategias y técnicas docentes: proporcionar mucha información, grabar la clase o señales sonoras.

- Organización del aula en función de la iluminación y que tenga buena sonoridad.

- Planteamiento de la clase: con un orden y considerando diversas partes.

- A nivel de cuestiones técnicas del instrumento: ejercicios de mano derecha y notas tenidas, insistir en la sujeción correcta del violín y la toma de arco, reforzar la memoria muscular y nemotécnica, contacto físico con el alumno, tutor-guía ${ }^{4}$ o guías de arco, referencias para el diapasón o intensificar el trabajo del oído.

Pueden consultarse más ampliamente en la propia tesis doctoral (Sánchez, 2017), así como en diversos trabajos que durante la investigación se realizaron, revelando parte de los hallazgos que se iban recopilando o trabajando en áreas paralelas: el aprendizaje de la viola por parte de los ciegos (Sánchez, 2016), instrumento muy afín al violín en cuanto manejo técnico; algunas estrategias relevantes en el aula de violín con invidentes (Sánchez y Muñoz, 2014); y el trabajo de orquesta y grupos de música de cámara con estudiantes ciegos (Sánchez y Muñoz, 2018).

4 Dispositivo creado en la Universidad Nacional de Lanús en Argentina. 


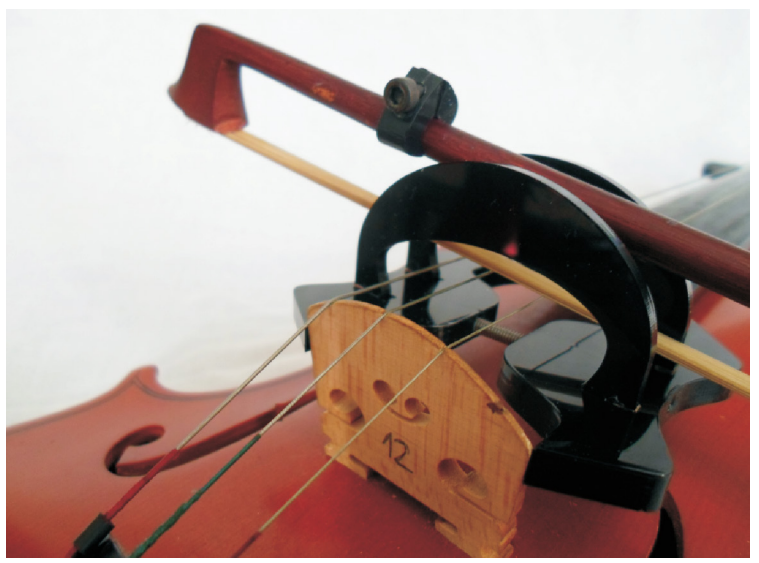

Figura 2. Paso del arco sobre las cuerdas usando el tutor-guía Fuente: fotografía facilitada por la profesora Lucía Patiño Mayer

Recogemos a continuación algunos testimonios que justifican algunas de ellas:

Desde mi niñez, he comenzado mi práctica diaria con notas tenidas en cuerdas al aire, usando arco completo y moviéndolo lentamente. Esto es tremendamente útil para tener un buen control del arco. También ayuda a desarrollar la habilidad de la escucha (...). Es necesario enseñar constantemente la manera correcta de sostener el violín y el arco (T. Wanami, entrevista, 07/01/2016).

Creo que adhiriendo pequeños círculos de metal muy delgado podría ayudar enormemente a la ubicación de los dedos en el diapasón del violín. Cada punto sería una altura. Esto para el ciego creo que sería muy fácil de procesar ya que con su tacto súper sensible sentiría la diferencia entre la madera y el metal (S. Aschero, entrevista, 09/09/2016).

El llevar la mano del ciego a que corrobore cómo es tal o cual postura o cómo pongo yo, su maestro, la postura de la cabeza o el hombro, puede ser mucho más importante de lo que creemos. Y obviamente, vital, a la hora de adquirir una correcta técnica (J. L. Cladera, entrevista, 01/10/2015).

- Actividades extraescolares: que puedan "tocar" y "experimentar", así como incluir algunas especiales a nivel curricular y para el desarrollo de valores.

- La clase colectiva, la práctica con el profesor pianista acompañante y las audiciones: ayudarse con la respiración, utilizar señales acústicas (en lugar de las visuales) y formación en paralelo (para reforzar la proximidad):

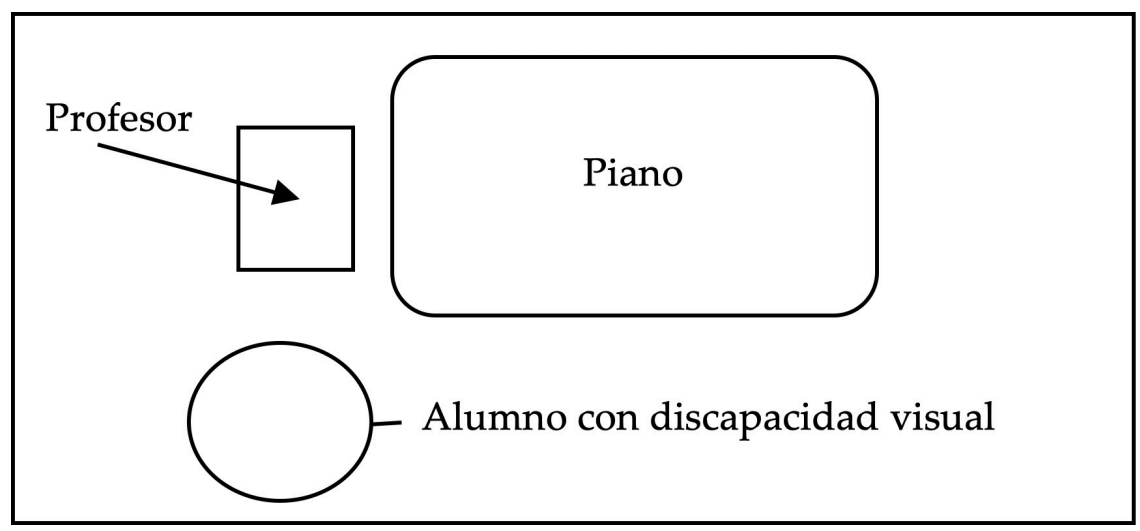

Figura 3. Formación en paralelo para las audiciones con el profesor pianista acompañante Fuente: elaboración propia

\subsection{Enseñanzas elementales: asignaturas de lenguaje musical y coro}

Para la asignatura de lenguaje musical durante las enseñanzas elementales, los recursos y técnicas recopiladas más destacadas fueron:

- A nivel de organización del aula que haya un orden estable, pasillos amplios para facilitar la movilidad, emplear sillas y mesas tradicionales o situar estratégicamente al alumno. 
- En cuanto a recursos materiales, usar gomets, emplear el Método Solfabra (García y Poveda, 2008), aplicar variables gráficas en las lecciones y ejercicios o usar libreta pentagramada adaptada.

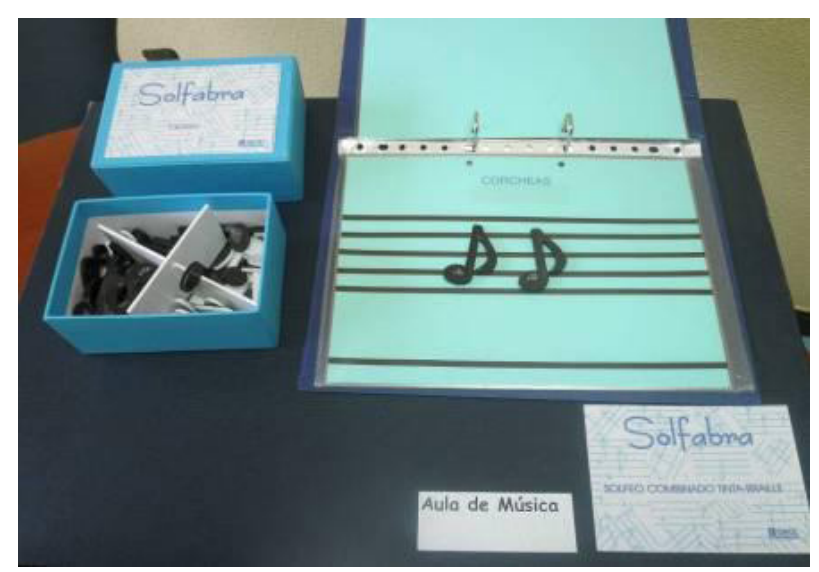

Figura 4. Método Solfabra

Fuente: elaboración propia

- A nivel de estrategias docentes, proporcionar al alumno un modelo táctil, modelar en su propio cuerpo los movimientos, conceder más tiempo para la realización de los exámenes o adaptar metodologías con señales visuales, como justifican los siguientes testimonios:

Las fotocopias de los exámenes se ampliaban para que los pudiera leer más fácilmente y, si era necesario, se me daba algo más de tiempo para realizarlos (A. Zamora, entrevista, 13/08/2015).

Para la práctica instrumental, no hay material adaptado, hay que adaptar las técnicas. Por ejemplo, en el xilófono, no se usan las dos manos, solamente una, y la otra se queda como referencia en el instrumento (M. M. López, entrevista, 29/09/2015).

- Y por último algunos aspectos concretos del lenguaje musical como marcar el pulso con el pie en las lecciones para evitar aprenderlas de memoria, sílabas rítmicas y canciones populares (conectando así con la metodología de Kodály), tener en cuenta que en braille se aprende primero las notas en corcheas, asignar números a cada fórmula rítmica para su trabajo colectivo o educar y estimular el oído, como recogimos de estos testimonios:

Para trabajar por ejemplo las fórmulas rítmicas colectivamente, asigno un número a cada una de ellas. Mientras el alumno vidente lo ha visto en la pizarra, el invidente lo ha memorizado. Sólo con decir el número todos lo recitan a la vez, trabajando así la fórmula rítmica que necesitemos en ese momento (I. Pulido, entrevista, 22/09/2015).

A menudo se dice que las personas ciegas tienen buenos oídos. Pero esto se debe a que entrenan a sus oídos de forma natural con el fin de vivir sin información visual. Si un alumno o alumna quiere ser músico, la formación para desarrollar el oído musical es esencial (T. Wanami, entrevista, 07/01/2016).

En la materia de coro en la etapa elemental se consiguieron estas estrategias:

- En cuanto al profesor y a la organización interna del coro, compaginar las indicaciones visuales con señales acústicas, estar más cerca de los coralistas de lo habitual o ayudarse con la respiración.

- A nivel de técnicas y estrategias para las clases de coro, cantar las notas o la letra para indicar pasajes o compases concretos, entregar con antelación las partituras, realizar exámenes parciales para que el repertorio a memorizar no sea muy extenso, desarrollar la concentración o que el alumno conozca la partitura de las otras voces.

- Ante dificultades rítmicas, algunos procedimientos son: hacer todo muy medido y contado, en las entradas exagerar las respiraciones o realizar un "compás de clavo", en los finales establecer una duración concreta de la nota final o utilizar algún tipo de chasquido, en los calderones especificar unos pulsos adicionales, en los cortes sin medida concreta que entren solo los videntes o hacerlos muy medidos para que entren todos, y cantar con el coro en pasajes de especial dificultad o para recalcar alguna cuestión de articulación, etc. Ello pudimos apreciarlo con los siguientes testimonios:

Cuesta mucho. Lo trabajamos mucho en los ensayos, y lógicamente no se puede tocar de una forma suelta, ad libitum, pues en ese caso ellos no saben entrar. Tiene que hacerse todo muy medido y contado (R. Romero, entrevista, 10/05/2015). 
En ocasiones, les pido a todos que cierren los ojos (videntes incluidos), y con una obra que tengan dominada, les pido que empiecen a cantar cuando quieran. Ellos mismos, con su propia respiración, si se concentran, logran empezar a cantar juntos sin necesidad de mi entrada (J. Santalices, entrevista, 28/11/2016).

En el caso de estos cortes sin medida concreta lo que hago es que a la hora de entrar, solo entran los videntes. A veces los hacemos muy medidos para que puedan entrar todos. Por ejemplo, contamos dos pulsos después de parar en un calderón y así entramos todos. Pero este sistema resulta muy mecánico para el que escucha (R. Romero, entrevista, 10/05/2015).

- En cuanto a los recursos materiales del aula, tener en cuenta la iluminación, organizar el aula y la colocación para que contribuya a potenciar el resto visual del alumno, hacer anotaciones con colores, bolígrafo o rotulador, y en el caso de alumnado con ceguera total usar grabadora de bolsillo, teléfono móvil o metrónomos afinadores para las anotaciones.

- En relación a las audiciones, algunos consejos son: colocar al grupo muy junto (sobre todo en acústicas complicadas), enseñar al alumno a moverse por el espacio (escena), para las danzas proporcionar un modelo, así como experimentar los movimientos lentamente o realizar un ensayo previo en la sala del concierto.

\subsection{Pruebas de acceso a enseñanzas profesionales}

Para las pruebas de acceso a estas enseñanzas, se estimó que en la prueba de instrumento (en este caso violín, pero extrapolable también a otra especialidad) era preciso realizar una adaptación curricular en la prueba de primera vista, ante la imposibilidad por parte del alumno con ceguera total. Por igual motivo, la prueba de piano complementario a primera vista ${ }^{5}$, se podría sustituir por la interpretación de un programa de tres piezas de un nivel acorde al curso.

Para las asignaturas de perfil teórico-musical, en este caso lenguaje musical, (o armonía, análisis e historia de la música si el acceso es a un curso más avanzado), se especificaron estos tres recursos:

- Adecuar el tamaño de los ejercicios si el alumno posee resto visual.

- Presentar las láminas y partituras en sistema braille si el alumno tiene ceguera total.

- Dejar al alumno un poco más de tiempo para realizar las pruebas.

\subsection{Enseñanzas profesionales: asignatura de violín}

Entrando ya en las asignaturas que comprenden las enseñanzas profesionales, en violín, se estableció, en primer lugar, algunas diferencias con respecto a las enseñanzas elementales. Se concretaron algunos recursos que en estos cursos ya no tienen aparición, tales como que la presencia de los padres en las clases se reduce y es más puntual, determinadas estrategias y recursos como el Método Solfabra, los musicogramas, las guías de arco, el tutor-guía o las pegatinas en relieve ya no se utilizan, y la clase colectiva al no impartirse en estos cursos, las estrategias y recursos se enfocan en las asignaturas de violín y música de cámara.

Posteriormente, se profundizó en algunas estrategias y recursos específicos para los cursos de violín de enseñanzas profesionales. Algunos de los más relevantes son: realizar ejemplos con el instrumento, enseñar a estudiar, contacto muy estrecho con el alumno, favorecer la autonomía e independencia del estudiante, paciencia por parte del alumno, metodología participativa, usar colores y practicar una enseñanza flexible.

La escucha de la música durante la práctica instrumental es de gran ayuda para un alumno con discapacidad. Que el profesor interprete fragmentos de la pieza u obra para mostrar al alumno la manera de interpretar, el estilo musical o la visión que tiene de ella. Cuando estoy estudiando una partitura, me gusta ampliar las digitaciones y marcar los matices dinámicos con colores (A. Zamora, entrevista, 13/08/2015).

\subsection{Enseñanzas profesionales: asignaturas de lenguaje musical y piano complementario}

Con respecto a la asignatura de lenguaje musical, se eliminan los gomets y materiales en relieve o los exámenes teóricos orales. En cuanto a las prácticas eran: potenciar el trabajo de polirritmias, polimetrías, etc.; hacer hincapié en el orden de notas de las alteraciones (en braille la armadura no recoge los nombres) o; que no es necesario eliminar la práctica de lectura a primera vista, ya que el pulso se marca con el pie.

Entrando en la materia de piano complementario, las estrategias y técnicas más destacadas son utilizar gomets con la notación en braille para los inicios, emplear un atril sujeto a una pinza para facilitar la lectura en alumnado con resto visual, tener en cuenta la complejidad de la lectura en braille con acordes que contienen notas de valores desiguales, practicar con manos separadas, memorizar por frases y hacer sesiones cortas de estudio, y, por último, para

Matizar que esta prueba no se realiza para el acceso al primer curso, pero si se solicita para el acceso a otros cursos diferentes a primero. Igual ocurre con las pruebas de armonía, análisis e historia de la música que se concretan más adelante, ya que de acuerdo con la LOE-LOMCE, es posible acceder a cualquier curso sin haber superado los anteriores por medio de una prueba, en la que el candidato demuestre los conocimientos necesarios. 
los saltos y las notas distantes usar la técnica de arrastre, la memoria motriz, el traslado en curva o la memoria espacial.

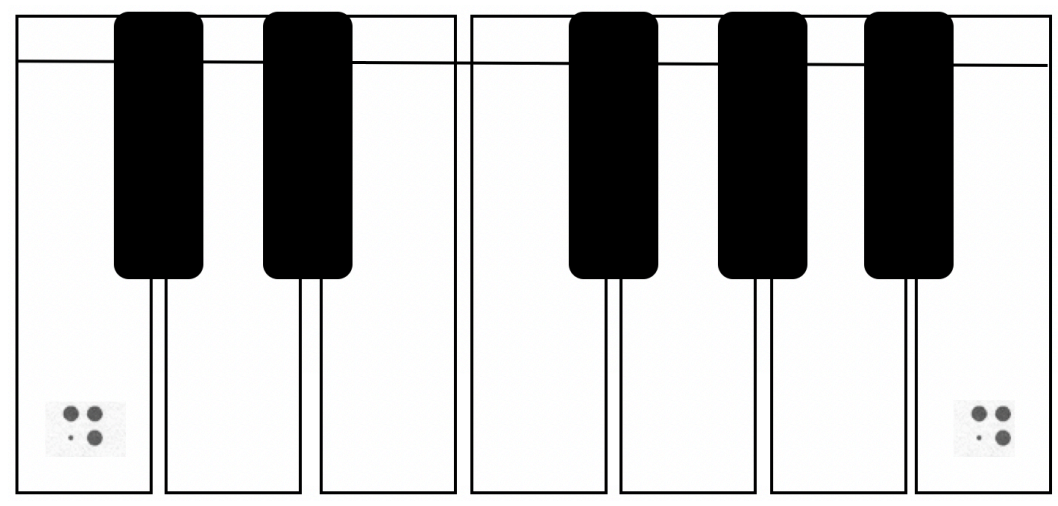

Figura 5. Colocación de los gomets con la notación braille en el teclado del piano Fuente: elaboración propia

\subsection{Enseñanzas profesionales: asignaturas de orquesta y música de cámara}

En las asignaturas de orquesta y música de cámara, a nivel del rol del profesor y la organización interna del grupo, se concretó compaginar las indicaciones visuales con señales acústicas, tener mucha sensibilidad, tratar al alumnado ciego de manera natural y contar con la ayuda del compañero de atril de la orquesta, como así reflejan en las entrevistas:

El director les habla, y marca el ritmo con un palo o aplaudiendo sus manos (A. Fikry, entrevista, 17/06/2015).

Cuando el director cambiaba el arco o la expresión, mi compañero de atril me ayudaba a entender el cambio ( $\mathrm{T}$. Wanami, 07/01/2016).

Tiene que estar muy pendiente de él, sobre todo al principio. Y siempre que das una explicación fijarte que él también se ha enterado. Tienes que buscarle trucos para sus entradas. Son muchas cosas las que tienes que tener en la cabeza. Y sobre todo, tratarlo igual que a los demás, porque si no se ve desplazado (R. Romero, entrevista, 10/05/2015).

En cuanto a la práctica orquestal y de música de cámara, algunos de los consejos más relevantes son: mayor concreción y detalle en las explicaciones orales, desarrollar la concentración, silencio y disciplina, que el alumno conozca la partitura de los otros instrumentos, colocar al grupo muy junto (sobre todo en acústicas difíciles), considerar el número de obras de repertorio, ensayar todo con antelación, tocar en algunos momentos puntuales (sobre todo en la práctica de música de cámara) y realizar un ensayo previo en la sala de concierto, tal y como recogimos de estas entrevistas:

Habrá que entender las dificultades de estos alumnos respecto a la lectura y memorización de las partituras, por lo que a este respecto, recomendamos considerar el número de obras del repertorio, así como reducir al máximo las piezas meramente técnicas, que supone un gran esfuerzo de memorización (Especialista anónimo, entrevista, 21/09/2016).

Si un determinado pasaje lo hemos ensayado forte, en el concierto no tengo la posibilidad de cambiarlo a otra dinámica ya que con mis gestos, no todos van a reaccionar del mismo modo (J. Santalices, entrevista, 28/11/2016). A la hora del concierto, se puede recordar alguna dinámica, con alguna señal, cantando o con la respiración (Director anónimo, entrevista, 01/02/2016).

En cuanto a las dificultades rítmicas que se producen en estos grupos, se concretaron diversas recomendaciones:

- Entradas: exagerar las respiraciones, realizar un "compás de clavo" o marcar alguna señal acústica.

- Cortes sin medida concreta: que entren solo los alumnos videntes, hacerlos muy medidos para que entren todos o pautarlos.

- Calderones: concretar unos pulsos adicionales.

- Cantar o emplear alguna consigna rítmica: para enfatizar cuestiones de articulación, etc.

Diversos testimonios, como el que se presenta a continuación respaldan estos planteamientos: 
En los cortes se dan muchas maneras, según sea el matiz, según sea con calderón, según se pueda pautar en cantidad de pulsos o tiempos, hay variadas formas. Se puede golpear cortito y veloz dos veces, se puede soplar, se puede hacer un gesto sonoro " $x$ ", el que se le ocurra a uno en el momento, y funciona (...). En situaciones que los matices generales o el ambiente o textura particular de la obra, la respiración, el soplido, un leve canturreo casi inaudible para otros permite que el músico ciego perciba el deseo del director de agregar un plus a lo dicho en la parte escrita. En otras, el énfasis en el golpe de batuta dice muchísimo (J. L. Cladera, entrevista, 01/10/2015).

Por último, se plasmaron diversos recursos materiales que son necesarios para el desarrollo de estas asignaturas, algunos de ellos son:

- Para alumnado con ceguera total: grabadora de bolsillo, teléfono móvil o metrónomos-afinadores, para las anotaciones.

- Para alumnado con resto visual: tableta digitalizadora y partituras en formato PDF, anotaciones con colores, bolígrafo o rotulador de trazo ancho y partituras en A3 o atril auxiliar.

\subsection{Enseñanzas profesionales: asignaturas de armonía, análisis e historia de la música}

Por último, en las asignaturas de armonía, análisis e historia de la música, se recogieron consejos para la organización de las aulas, tanto para la atención de estudiantes con ceguera total como con resto visual, y se profundizó en estrategias docentes y recursos materiales que son precisos. Algunos de los más relevantes son: la máquina perkins y el sistema braille, realizar ejemplos al piano por parte del profesor y utilizar el programa informático BME, tener en cuenta que el alumno con ceguera total necesita un mayor estudio para el análisis (al ser la lectura braille secuencial y lineal), utilizar para el análisis el formato "compás sobre compás” que resulta más adecuado, considerar que la corrección de exámenes y ejercicios es más lenta, y emplear la repetición de apuntes, el libro hablado o la grabadora.

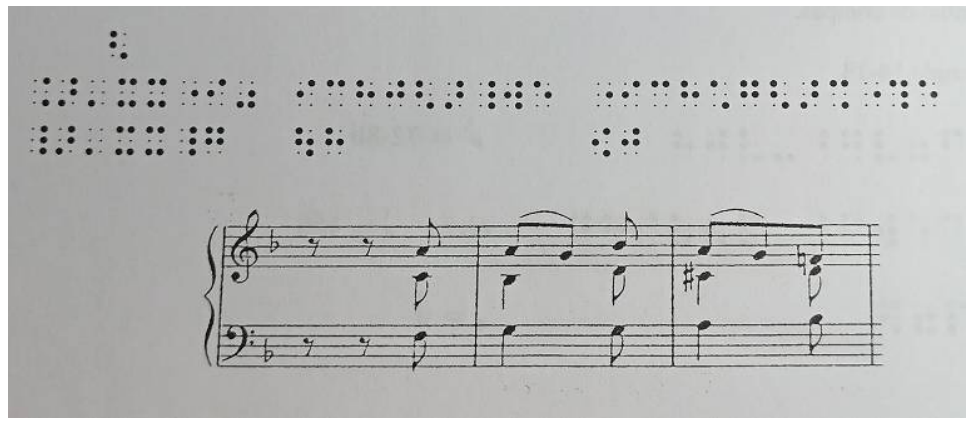

Figura 6. Fragmento en formato "compás sobre compás” que resulta oportuno para el análisis musical Fuente: Aller (2001, p. 104)

Algunos de estos testimonios respaldan estos planteamientos:

Escribía en la máquina perkins mis ejercicios, y luego se los leía a mi profesora. En los exámenes los enviaba para transcribir a tinta (C. Fernández, entrevista, 19/01/2017).

En mi aprendizaje musical de la armonía y el análisis musical, he utilizado las herramientas que se citan anteriormente, el braille, las transcripciones y el BME. Aunque también he de decir que he trabajado mucho auditivamente, y también ciertos profesores me ponían ejemplos tocando en el piano todo lo que era necesario, lo que facilita bastante el trabajo para nosotros (M. Rojas, entrevista, 01/02/2017).

\section{Análisis y evaluación de los resultados}

Para analizar y valorar los resultados obtenidos, la investigación consideró dos ejes: por un lado, el análisis y evaluación de los expertos, y por otro, un análisis reflexivo a partir del diálogo de saberes.

El análisis y la evaluación de los expertos arrojó resultados positivos. Estos profesionales valoraron, entre otros aspectos, el rigor, realismo, interés, utilidad, claridad, los diferentes puntos de vista de los entrevistados y que es un material muy completo, así como recomendaban este material pedagógico a los profesores, ya que estiman que favorecería una educación inclusiva.

Entre las propuestas y planteamientos que se abordan de manera más satisfactoria, efectiva y acertada, consideraron que los aspectos metodológicos en cuanto a la actitud del profesor ante el alumno con discapacidad visual, 
las aportaciones en la práctica grupal, la minuciosidad, el gran número de estrategias y recursos, la profundidad y realismo en todas las cuestiones, los planteamientos para las cuestiones técnicas del violín, etc.

Las propuestas y sugerencias de estos expertos se organizaron en cuatro apartados, cambios que se sugieren, dificultades, matices e incorporaciones y sugerencias:

- Cambios que se sugieren: el Braille'n Speak, que actualmente está en desuso. Es más adecuado la grabadora, el móvil o anotadores como la línea braille focus. También los gomets en el teclado del piano, más adecuado hacer la ubicación de las notas a través de las teclas negras. O a nivel de los exámenes teóricos orales en lenguaje musical en las EE.EE., mejor realizarlos con la máquina perkins o el ordenador, aunque en algunos casos, se pueden seguir haciendo incluso en las EE.PP.

- Dificultades que se aprecian: el trabajo con instrumentos de láminas (baquetas) y que los docentes aprendan braille.

- Matizaciones e incorporaciones que se proponen: en referencia al programa informático BME, destacar que puede hacer anotaciones en las partituras digitalizadas. Sobre la lectura a primera vista en lenguaje musical y coro, matizar que sí es posible y recomendada, pero si el alumno cuenta con más tiempo de preparación. O al señalar el mayor tiempo para realizar los exámenes, concretar que no es una opción, sino algo imprescindible.

- Sugerencias y aportaciones: dedicar tiempo a aprender los nuevos signos musicales y fomentar el programa BME2; el cansancio que presentan estos estudiantes puede entrenarse y poder considerar clases de refuerzo; en coro, es interesante que el niño disponga de partitura o guión para consulta; o que pueda incluirse en el currículo de las enseñanzas profesionales algunas materias optativas sobre la técnica Alexander o Feldenkrais, así como otra relacionada con el miedo escénico.

Asimismo, con respecto a la evolución de la herramienta inclusiva que se consideró y que cristalizó finalmente en un compendio de buenas prácticas, consideramos que fue positivo, pues la investigación fue adaptándose a la realidad de la situación que encontramos, tratando de ofrecer la mejor solución y respuesta.

En cuanto al segundo de los objetivos básicos que se mencionó en la introducción, "elaborar alguna herramienta o método que ayudara en esta labor a los docentes de conservatorio", se consideró que el Compendio de Buenas Prácticas, Recursos y Estrategias Metodológicas respondía a este cometido, y, por tanto se cumplió este objetivo, así como el primero, pues a través de él, el profesorado de estos centros podría resolver sus dudas sobre la enseñanza de estos estudiantes.

Por último, el aprendizaje y servicio ha sido una cuestión que se ha conseguido, en la relación de estos espacios de reflexión que siguen abiertos y en continua reflexión de la praxis-teoría, y en la devolución que también se ha realizado del compendio elaborado a docentes de la música que se involucraron en esta investigación.

\section{Conclusiones}

Finalmente, tras la evaluación de los expertos y este análisis reflexivo, la investigación concretó las siguientes conclusiones.

Las evaluaciones fueron positivas, se estimó que los planteamientos eran fiables, viables y sólidos, se daba con ellos respuesta a los problemas y dificultades que el alumnado de música ciego tiene y se habían integrado dispositivos y herramientas de reciente creación que se conocieron en el trabajo de campo, entre otros aspectos.

El alumnado con discapacidad visual no precisa un repertorio o método concreto distinto al de los estudiantes con visión. También que la discapacidad no debe suponer una barrera en la enseñanza.

Por otro lado, con esta investigación, se contribuye en un campo en el cual los docentes suelen carecer de información, están desorientados o no disponen de formación previa (Chaves et al., 2015; Chávez, 2010; Dias, 2010).

Atendiendo al contenido del trabajo, se ha actuado en profundidad desde diferentes ámbitos, y se ha buscado flexibilidad y versatilidad en los planteamientos, para que el docente escoja aquellos más adecuados para su alumno.

Asimismo, el compendio no es un documento cerrado, pues se desea seguir investigando y poder ir integrando futuras aportaciones a nivel didáctico o de recursos (evolución en la tiflotecnología), o puede ampliarse para otras especialidades instrumentales. Además, muchos planteamientos son trasladables al alumnado de violín con otras capacidades o videntes, como refleja el siguiente testimonio:

Lo que en un principio fue una medida extraordinaria para atender a un alumno (invidente), ya lo utilizan todos mis alumnos en el primer curso de viola. Ahora, después de verlo los profesores de violín, también han decidido implantarlo para sus pupilos (A. Pérez, entrevista, 06/02/2017).

Además, el compendio puede ser también útil para directores de agrupaciones musicales que integren ciegos, para profesores de especialidades instrumentales afines, y para los especialistas del resto de asignaturas que componen estas enseñanzas, como expuso uno de los expertos en su evaluación: “(Este trabajo) creo será de gran 
utilidad a distintos profesionales, tanto de violín, como de otras materias" (Especialista anónimo, evaluación, 02/05/2017).

Asimismo, una aportación interesante que la investigación deja es que los estudios superiores de música, en la especialidad de dirección, pudieran incorporar alguna asignatura optativa, masterclass o jornadas. De hecho, siguiendo esta línea, también sería valioso que los estudios superiores de música en la especialidad de pedagogía, pudieran incorporar alguna asignatura específica de atención a la diversidad: "Me ha dado mucha pedagogía a la hora de tratar a otras agrupaciones de no ciegos, incluso a los alumnos en los colegios normales, sean ciegos o no" (R. Romero, entrevista, 10/05/2015).

A nivel personal, la etnografía reflexiva y colaborativa, nos ha dejado también un valioso aprendizaje tanto a nivel docente, como humano y personal. Este parecer lo compartía uno de los expertos en su evaluación:

Da la sensación que cuando se aborda la inclusión, quien se beneficia es el alumno con déficit visual parcial o total, pero la experiencia de años me dice que los verdaderamente beneficiados somos el resto: profesores, alumnos y en general todo el sistema educativo. La divergencia es una apuesta para aprender con corazón y un estímulo para la inteligencia (M. J. G., evaluación, 08/05/2017).

Por otro lado, una reflexión relevante de este trabajo, ha sido la importancia de la riqueza de la diversidad y el derecho a ser diferentes, pues como expresan Espinosa, Motos, Valdivieso y Poyatos (2000, p. 32) "los humanos somos diferentes desde todo punto de vista".

Además, se tiene previsto realizar en próximos meses una Guía didáctica con una síntesis del compendio, para acercar estas estrategias educativas a las familias y a los docentes de alumnado con discapacidad visual.

También consideramos enriquecedor potenciar las orquestas con diversidad, así seguir una línea similar a la Orquesta de la Luz y la Esperanza de Egipto de la Asociación "Al Nour Wal Amal”, la cual es la única orquesta sinfónica en el mundo donde todas sus componentes poseen discapacidad visual. $\mathrm{Y}$ en particular potenciar los grupos musicales de personas con discapacidad visual, tratando de retomar, si es posible, la actividad de aquellos que dejaron de funcionar, lo que permite favorecer así, a un mayor número de estas agrupaciones, tal y como había hace años y se señaló en el apartado 1.

Y por último, el trabajo colaborativo y el diálogo de saberes han sido muy fructíferos en el proceso de la etnografia. Cada participante de la investigación (alumnado, profesorado, familias y expertos) ha aportado experiencias, actitudes y aspectos relevantes en la formación con alumnado de discapacidad visual que puede trasladarse a la praxis de una educación inclusiva.

\section{Referencias bibliográficas}

Abramo, J. M. y Pierce, A. E. (2013). An ethnographic case study of music learning at a school for the blind. Bulletin of the Council for Research in Music Education, 195, 9-24. https://doi.org/10.5406/bulcouresmusedu.195.0009

Acero, L. (2010). Un día en clase: reflexiones de una maestra. Integración, 58, (Ed. Digital). http://repositoriocdpd.net:8080/ bitstream/handle/123456789/1605/Art_AceroArranzL_Undiaenclase_2010.pdf? sequence=1

Aguilar, S. y Barroso, J. (2015). La triangulación de datos como estrategia en investigación educativa. Píxel-Bit. Revista de Medios y Educación, 47, 73-88. https://www.redalyc.org/pdf/368/36841180005.pdf

Albertí, M. y Romero, L. (2010). Alumnado con discapacidad visual. Barcelona: Graó.

Aller, J. (2001). Manual simplificado de musicografia braille. Madrid: Organización Nacional de Ciegos Españoles (ONCE).

Arias, M. M. (2000). La triangulación metodológica: sus principios, alcances y limitaciones. Investigación y educación en enfermería, 18 (1), 13-26. https://www.redalyc.org/pdf/1052/105218294001.pdf

Arnaiz, P. (1994). Deficiencias visuales y psicomotricidad: teoría y práctica. Madrid: ONCE. Departamento de Servicios Sociales para Afiliados.

Barraga, N. (1985). Disminuidos visuales y aprendizaje: (enfoque evolutivo). Madrid: ONCE.

Betrián, E., Galitó, N., García, N., Jové, G. y Macarulla, M. (2013). La triangulación múltiple como estrategia metodológica. Revista Iberoamericana sobre Calidad, Eficacia y Cambio en Educación (REICE), 11 (4), 5-24. https://www.redalyc.org/ pdf/551/55128238001.pdf

Cabero, J. y Llorente, M. C. (2013). La aplicación del juicio de experto como técnica de evaluación de las tecnologías de la información (TIC). Eduweb, 7 (2), 11-22. http://servicio.bc.uc.edu.ve/educacion/eduweb/v7n2/art01.pdf

Campo, L. (2008). El aprendizaje servicio en la universidad como propuesta pedagógica. En M. Martínez (ed.), Aprendizaje servicio y responsabilidad social de las universidades (pp. 81-91). Barcelona: Octaedro.

Chaves, A., Godall, P. y Zattera, V. (2015). La enseñanza de la musicografía braille: consideraciones sobre la importancia de la escritura musical en Braille y la transcripción de materiales didácticos. Revista da ABEM, 23 (34), 138-151. http://www.abemeducacaomusical.com.br/revistas/revistaABEM/index.php/revistaabem/article/viewFile/536/444

Chávez, P. G. (2010). Estrategias de estudio utilizadas por pianistas ciegos. En Actas de la IX Reunión de SACCoM (Sociedad Argentina para las Ciencias Cognitivas de la Música) (pp. 74-79). http://www.saccom.org.ar/2010_reunion9/actas/13.ChavezP.pdf 
Clark, A. y Murphy, F. (1999). Enseñanza de la música a estudiantes discapacitados visuales en el ámbito escolar convencional. Entre Dos Mundos, 10, 5-16.

Dias, I. C. (2010). La educación musical de personas con deficiencia visual y la musicografía braille: de la musicalización a la lectura y a la escritura de la partitura en braille. En Actas de la IX Reunión de SACCoM (Sociedad Argentina para las Ciencias Cognitivas de la Música) (pp. 58-64).

Dietz, G. (2009) Multiculturalism, Interculturality and Diversity in Education: an anthropo- logical approach. Muenster \& Nueva York: Waxmann.

Dietz, G. (2011). Hacia una etnografía doblemente reflexiva: una propuesta desde la antropología de la interculturalidad. Revista de Antropología Iberoamericana (AIBR), 6 (1), 3-26. https://doi.org/10.11156/aibr.060102e

Dietz, G. (2012). Reflexividad y diálogo en etnografía colaborativa: el acompañamiento etnográfico de una institución educativa “intercultural” mexicana. Revista de Antropología social, 21, 63-91. https://doi.org/10.5209/rev_RASO.2012.v21.40050

Díez, A. (1996). Agrupaciones artísticas. Congreso Estatal sobre Prestación de Servicios para Personas Ciegas y Deficientes Visuales, 2, pp. 263-268). Madrid: ONCE.

Donolo, D. S. (2009). Triangulación: procedimiento incorporado a nuevas metodologías de investigación. Revista Digital Universitaria, 10 (8). http://www.revista.unam.mx/vol.10/num8/art53/int53.htm

Escobar-Pérez, J. y Cuervo-Martínez, A. (2008). Validez de contenido y juicio de expertos: una aproximación a su utilización. Avances en medición, 6, 27-36.

Espejo, B. (1993). El braille en la escuela: una guía práctica para la enseñanza del braille. Madrid: ONCE.

Espinosa, F., Motos, I. M., Valdivieso, S. y Poyatos, E. (2000). En la información reside la tolerancia: una actividad de acercamiento al braille con niños videntes. Integración, 32, 32-41.

Folgueiras, P., Luna, E. y Puig, G. (2013). Aprendizaje y servicio: estudio del grado de satisfacción de estudiantes universitarios. Revista de educación, 362, 159-185. http://diposit.ub.edu/dspace/bitstream/2445/65896/1/586668.pdf

Fuentes, J. (1995). Método Alameda. Madrid: ONCE.

Garaialde, K., Laquidain, N. y Urbina, J. (1992). La diferencia como cualidad: la integración de una niña ciega en una clase ordinaria. Integración, 8, 29-41.

García, L. y Poveda, M. P. (2008). Solfabra. Explanada, 65, 18-21.

González, G., Martínez, M. A., Fernández, J., Soler, M. C. y Ruíz, S. (2000). La flauta mágica: ópera adaptada y actividades para alumnos de educación integrada. Integración, 32, 24-31.

Gorbunova, I. B. y Voronov, A. M. (2018). Music Computer Technologies in Computer Science and Music Studies at Schools for Children with Deep visual Impairment. In $16^{\text {th }}$ International Conference on Literature, Languages, Humanities and Social Sciences (LLHSS-18) of Budapest, pp. 15-18. https://doi.org/10.17758/URUAE4.UH10184021

Ley Orgánica 2/2006, de 3 de mayo, de Educación (BOE No106 del 04-05-2006). https://www.boe.es/boe/dias/2006/05/04/pdfs/ A17158-17207.pdf

Ley Orgánica 8/2013, de 9 de diciembre, para la mejora de la Calidad Educativa (BOE No295 del 10-12-2013). https://www.boe. es/boe/dias/2013/12/10/pdfs/BOE-A-2013-12886.pdf

Lucas, S., y Martínez, A. (2012). La implantación y difusión del Aprendizaje-Servicio en el contexto educativo español. Retos de futuro de una metodología de enseñanza-aprendizaje para promover la innovación en la Educación Superior. VII Congreso Internacional de Docencia Universitaria e Innovación. Barcelona: Universidad de Barcelona. http://www.cidui.org/revistacidui/index.php/cidui/article/download/122/110

Marcos, M. (1999). Percepción visual y ceguera. En M. R. Villalba (dir.) e I. Martínez (coord.), Aspectos evolutivos y educativos de la deficiencia visual (capítulo I, vol. I). Madrid: ONCE.

Mendía, R. (2012). El aprendizaje-servicio como una estrategia inclusiva para superar las barreras al aprendizaje y a la participación. Revista de Educación Inclusiva, 5 (1), 71-82. https://www.revistaeducacioninclusiva.es/index.php/REI/article/viewFile/222/216

Muñoz, P. (2012). Dilemas de una antropología comprometida: entre autores y experiencias etnográficas. En Peña, B. (eds.). Desarrollo Humano, 249-281. Madrid: Vision Libros.

Muñoz, P., Mateo, C. y Álvarez, M. M. (2014). Perfiles docentes a partir de una etnografía en la escuela. Investigación acción desde el Prácticum. Historia y Comunicación Social, 19, 363-374. https://doi.org/10.5209/rev_HICS.2014.v19.45139

Puig, J. M., Gijón, M., Martín, X. y Rubio, L. (2011). Aprendizaje-servicio y Educación para la Ciudadanía. Revista de educación, $\mathrm{N}^{\mathrm{o}}$ extraordinario, 45-67.

Quintero, F. (2000). "Tras la huella de don Quijote”: integrarse, participar, ser uno más. Integración, 33, 26-32.

Robles, P. y Rojas, M. C. (2015). La validación por juicio de expertos: dos investigaciones cualitativas en Lingüística aplicada. Revista Nebrija de Lingüistica Aplicada, 18, 124-139. http:/www.nebrija.com/revista-linguistica/files/revistasPDF/Revista\%20completa\%2018.pdf

Rodríguez, C., Pozo, T. y Gutiérrez, J. (2006). La triangulación analítica como recurso para la validación de estudios de encuesta recurrentes e investigaciones de réplica en Educación Superior. Revista Electrónica de Investigación y Evaluación Educativa (Relieve), 12 (2), 289-305. https://doi.org/10.7203/relieve.12.2.4231

Sánchez, L. (2016). Educación inclusiva con músicos invidentes: aprendizaje de la viola. Opción: Revista de Ciencias Humanas y Sociales, (10), 734-742. 
Sánchez, L. (2017). La didáctica del violín en alumnos con discapacidad visual. Compendio de Buenas Prácticas, recursos y estrategias metodológicas para una Educación Inclusiva en las Enseñanzas Elementales y Profesionales de Música. Universidad Católica San Antonio, Murcia. http://hdl.handle.net/10952/2589

Sánchez, L. y Muñoz, P. (2014). Estrategias metodológicas en el aula de violín para el alumnado con discapacidad visual. En Navarro, J., Gracia, M. D., Lineros, R. y Soto, F. J. (coords.). Claves para una educación diversa. Murcia: Consejería de Educación, Cultura y Universidades.

Sánchez, L. y Muñoz, P. (2018). El trabajo de orquesta y grupo de cámara con estudiantes ciegos. En P. Muñoz y M. J. Vitón (coord). Comunidad, desarrollo y escenarios educativos emancipatorios. Reflexionando la alteridad sociocultural (pp. 165171). Compobell.

Tapia, M. N. (2010). La propuesta pedagógica del "Aprendizaje-Servicio": una perspectiva latinoamericana. Tzhoecoen, Revista Cientifica, 5, 23-43.

Taylor, S. J. y Bogdan, R. (1987). Introducción a los métodos cualitativos de investigación. Barcelona: Paidós.

Tomás, B. y Barcelón, L. (2006). Asamblea de aula para todos: una adaptación para la inclusión de un alumno ciego. Integración, 49, 25-33.

Velasco, H. y Díaz de Rada, A. (1997). La lógica de la investigación etnográfica. Madrid: Trotta.

Vitón, M. J. (2012). Diálogos con Raquel: Praxis Pedagógicas y reflexión de saberes para el desarrollo educativo en la diversidad cultural. Madrid: Ed. Popular. https://doi.org/10.17141/iconos.50.2014.1438 